Marquette University
e-Publications@Marquette

Master's Theses (2009 -)

Dissertations, Theses, and Professional

Projects

\title{
Root-End Surgery or Nonsurgical Retreatment: Are There Differences in Long Term Outcome?
}

Enida Haxhia

Marquette University

Follow this and additional works at: https://epublications.marquette.edu/theses_open

Part of the Dentistry Commons

\section{Recommended Citation}

Haxhia, Enida, "Root-End Surgery or Nonsurgical Retreatment: Are There Differences in Long Term Outcome?" (2021). Master's Theses (2009 -). 651.

https://epublications.marquette.edu/theses_open/651 
ROOT-END SURGERY OR NONSURGICAL RETREATMENT:

ARE THERE DIFFERENCES IN LONG-TERM OUTCOME?

\author{
by \\ Enida Haxhia, D.D.S.
}

A Thesis Submitted to the Faculty of the Graduate School, Marquette University, in Partial Fulfillment of the Requirements for the Degree of Master of Endodontics

Milwaukee, Wisconsin

May 2021 


\title{
ABSTRACT \\ ROOT-END SURGERY OR NONSURGICAL RETREATMENT: \\ ARE THERE DIFFERENCES IN LONG-TERM OUTCOME?
}

\author{
Enida Haxhia, D.D.S
}

Marquette University, 2021

INTRODUCTION: The decision of which modality of secondary endodontic treatment to perform is multifactorial and clinician dependent. Literature surrounding the long-term survival of nonsurgical retreatment compared to root-end surgery remain equivocal and warrant further investigation. This 7-year retrospective study seeks to compare the outcome of nonsurgical retreatments with that of root-end surgeries performed on teeth without prior nonsurgical retreatments.

METHODS: Insurance claims from 1021 teeth of 987 patients in the Delta Dental of Wisconsin database were analyzed from the years 2008-2017. Tooth survival was evaluated using Cox regression models and p-value was set at 0.05. Survival time was considered from the time of completion of nonsurgical retreatment or root-end surgery to time of an untoward event, defined as extraction after root-end surgery or extraction/rootend surgery after nonsurgical retreatment. Only procedures performed by endodontists were included in the analysis.

RESULTS: The survival rate of teeth that received nonsurgical retreatment was $90 \%$ after 2 years, $86.8 \%$ after 4 years and $85 \%$ after 6 years. The survival rate of teeth that received root-end surgery was $93.7 \%$ after 2 years, $90.5 \%$ after 4 years and $88 \%$ after 6 years. No statistically significant difference was found in survival of nonsurgical retreatment compared to root-end surgery. Likewise, no statistically significant difference was found within or between tooth types (anterior, premolar, molar) when comparing nonsurgical retreatment to root-end surgery.

CONCLUSION: The results of this study indicate that clinicians can choose either nonsurgical retreatment or root-end surgery after failed primary root canal therapy. Tooth location was not a determining factor in the survival rate after nonsurgical retreatment or root-end surgery. 


\title{
ACKNOWLEDGEMENTS
}

\author{
Enida Haxhia, D.D.S.
}

I am extremely grateful to Marquette University School of Dentistry and the Endodontics department for giving me the opportunity to pursue my goal of becoming an endodontist. My time here has been one of the most rewarding experiences of my life.

I would like to thank my committee members, Dr. Mohamed Ibrahim and Dr. Pradeep Bhagavatula, for their help and guidance in completing this project.

Dr. Ibrahim, I have appreciated your influence as a mentor, but more so, as a friend during my time in the program and will reflect fondly on our experiences together.

I would also like to thank the other amazing faculty in our department, Dr. Joseph Gaffney and Dr. Joseph DeGuzman. Your mentorship and support have been invaluable, and I cannot thank each of you enough for your dedication to this program.

A sincere appreciation specifically goes to Dr. Lance Hashimoto. Your outstanding clinical skills, your patience, your kindness and your generosity are unmatched. It has been a complete joy to work under your guidance. You are exceptional! I will be forever grateful for knowing you!

The two people who I am lucky to call my coresidents, Dr. Lauren Loney and Dr. Tom Korte, you have been instrumental to my experience here at Marquette. I could not have asked for better, more compatible, or more respectful coresidents to share this journey with. I have truly cherished our camaraderie over the last two years and look forward to our lifelong friendship that will follow.

Additionally, I would like to acknowledge two of my other coresidents. Dr. Mike Smoljan for all his advice and leadership during the first year of my residency, and Dr. Andrea Tsatalis for her close friendship throughout my second year.

Finally, I would like to thank my family. I would not be where I am today without their sacrifices, love, support and encouragement. I hope to have made them proud. 


\section{TABLE OF CONTENTS}

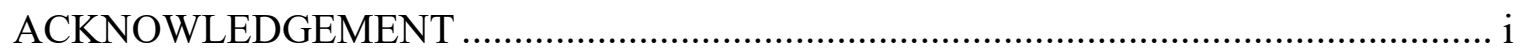

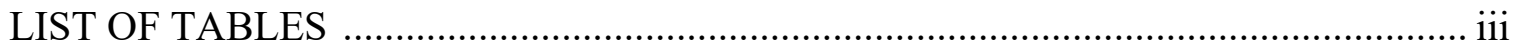

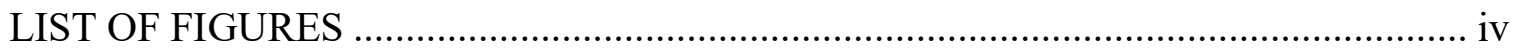

INTRODUCTON

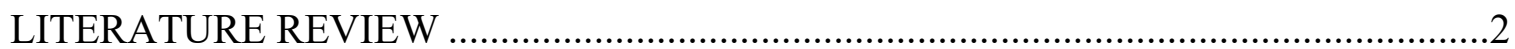

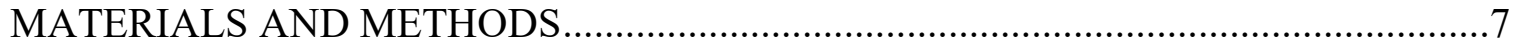

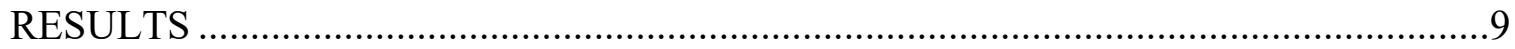

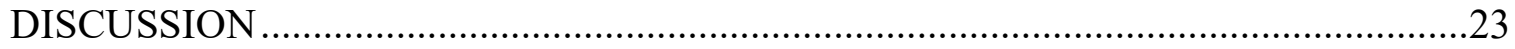

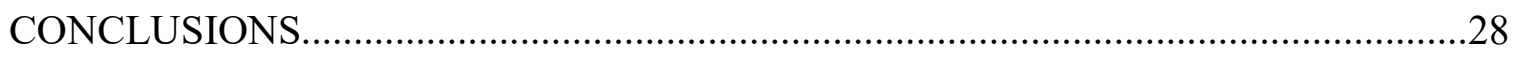

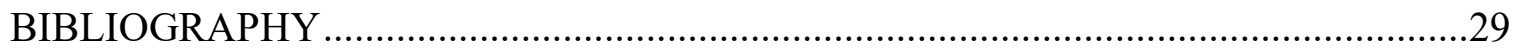




\section{LIST OF TABLES}

Table 1: Population Data Stratified by Retreatment Type..............................................11

Table 2: Survival Rates for Nonsurgical Retreatment and Root-End Surgery .................13

Table 3: Nonsurgical Retreatment Survival Rate by Tooth Type...................................15

Table 4: Pairwise Tooth Type Comparison for Nonsurgical Retreatment .......................15

Table 5: Nonsurgical Retreatment Survival Rates for Different Age Groups ..................17

Table 6: Pairwise Comparison of Age Groups for Nonsurgical Retreatment ..................18

Table 7: Root-End Surgery Survival Rate by Tooth Type ...........................................19

Table 8: Pairwise Tooth Type Comparison for Root-End Surgery ................................20

Table 9: Root-End Surgery Survival Rates for Different Age Groups ...........................21

Table 10: Pairwise Comparison of Age Groups for Root-End Surgery ............................22 


\section{LIST OF FIGURES}

Figure 1: Comparison of Tooth Survival Between Nonsurgical Retreatment and Root-End

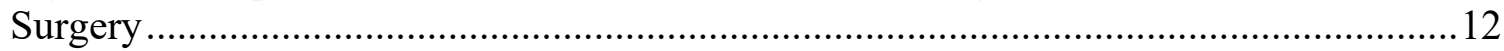

Figure 2: Nonsurgical Retreatment Outcomes Categorized by Tooth Location...............13

Figure 3: Nonsurgical Retreatment Outcomes Categorized by Age Groups ....................14

Figure 4: Root-End Surgery Outcomes Categorized by Tooth Location ........................15

Figure 5: Root-End Surgery Outcomes Categorized by Age Groups ............................16 


\section{INTRODUCTION}

Nonsurgical root canal therapy (NSRCT) is a procedure that has historically yielded predictable outcomes. Longitudinal epidemiologic studies by Salehrabi and Lazarski have found the retention rates of primary endodontic therapy to range between 94\%-97\% (1,2). A multitude of factors have been examined impacting clinical success. These factors include, but are not limited to, following proper aseptic and disinfection protocols(3), presence or absence of apical periodontitis(4), timing of core and/or post placement(5), adequate coronal restoration(6), and provider training(7). Other factors such as single visit versus multiple visit treatments have not been found to exhibit a significant difference in regard to healing or success rate $(8,9)$.

If NSRCT fails, the next course of treatment can comprise of nonsurgical retreatment or root-end surgery — providing that the tooth is deemed restorable and periodontally sound. Selection between these two treatment modalities necessitates clinician familiarity with long-term outcome and survival rates of teeth subjected to secondary endodontic therapy. Favorable outcomes have been reported for surgical(10) and nonsurgical retreatments(11); however, data remains inconclusive regarding their long-term outlook. Current literature lacks a definitive consensus on selecting nonsurgical retreatment versus root-end surgery when a tooth presents with persistent periapical pathosis and/or clinical symptoms after NSRCT. Additionally, extensive variability exists in study designs, methodology, clinical protocols and length of observation for nonsurgical retreatment and root-end surgery studies. 


\section{LITERATURE REVIEW}

\section{Outcomes of Root-End Surgery}

Regarding endodontic microsurgery, different types of studies have been conducted over the years reporting variable success rates. A prospective clinical and radiographic study evaluation by von Arx et al(12) reported $83.8 \%$ healing or success 1 year after periapical surgery. Another prospective study of similar nature but with a follow up ranging from 1 to 4 years showed a higher success rate of 91.2\%(13).

The utilization of modern techniques and armamentarium have led to a significant improvement in success of root-end surgery. Results from the meta-analysis by Tsesis et al(14) concluded an outcome of $91.6 \%$ success for teeth treated with a modern surgical technique in a follow up of at least 1 year. This is a stark contrast from the $44.2 \%$ success found in teeth that were surgerized using the traditional techniques(15). The differences between traditional and modern methods include the switch from a 45-degree bevel root end resection to a zero or minimal degree bevel, as well as the shift from using burs for retrograde root end preparation to utilizing ultrasonic tips under high magnification(16). These changes along with the introduction of more biocompatible root end filling materials(17) have allowed for root-end surgery to yield highly predictable results.

Various other studies have confirmed the improved surgical results. Rubinstein et al(18) found $96.8 \%$ radiographic success and reported an average healing time of lesion consisting of 7.2 months. The same author subsequently followed these healed cases for 5-7 years and validated the long-term success of apical microsurgery with a rate of $91.5 \%$ from his second study(19). Another long term follow up report by Song et al(10) reported a success rate of $93.3 \%$ after a period of 10 years. The probability of success of modern 
root-end surgery procedures appears to be consistently high given the results of these outcome studies.

\section{Outcomes of Nonsurgical Retreatment}

Similar to root-end surgery studies, the same observation can be made for studies involving nonsurgical retreatment. They vary in methodology of study, clinical technique and length of follow up. The largest epidemiologic evaluation on the outcome of nonsurgical retreatment conducted by Salehrabi et al(11) concluded that $89 \%$ of teeth were retained and functional in the oral cavity 5 years after the procedure. That study observed 4744 teeth that had nonsurgical retreatment performed by endodontists without specification of techniques utilized, tooth location or etiology of previous root canal failure. The distinction of having these procedures performed by only endodontists was important since in other studies(20) teeth have been treated by either general dentists or in school settings.

Likewise, the prospective cohort Toronto study looking at the outcome of nonsurgical retreatment with a 4-6 years follow up concluded similar results to Salehrabi et al. Farzaneh et al(20) reported an overall success rate of $81 \%$ based on clinical and radiographic evaluations, but a 93\% retention or survival rate for teeth that were still asymptomatic and functional. Unlike Salehrabi et al, the procedures in the Toronto study were performed by graduate students.

Gorni et al(21) distinguished success of nonsurgical retreatment based on whether or not the root canal morphology was altered from the prior endodontic treatment. This study found $86.8 \%$ of success for the non-altered group versus a $47 \%$ success for the previously altered group in a 2 year follow up, with an overall success of $69.03 \%$. These 
results portray the variability of cases which undergo nonsurgical retreatment, which in turn may affect and relate to the outcome.

Older studies such as the one by Sundqvist et al(22), reporting a $74 \%$ success rate, have recognized the negative influence of the gram positive microorganism E. faecalis and the poorer prognosis associated with a larger size periapical lesion in the outcome of nonsurgical retreatment. The study by Chugal et al(23) substantiated the previous finding, stating that a larger periapical pathology and bacterial involvement will negatively impact the outcome of endodontic therapy. These factors continue to play a role and may explain the difference in outcome between initial therapy and nonsurgical retreatment. The retrospective study by Imura et al(24) found a $85.9 \%$ success in nonsurgical retreatment compared to a $94 \%$ success in initial endodontic therapy in teeth treated solely by specialists.

\section{Studies Directly Comparing Outcomes of Nonsurgical Retreatment to Root-End Surgery}

Some authors have conducted studies that directly compared nonsurgical retreatment with endodontic surgery. Kang et al(25) reported overall success rates of $92 \%$ for endodontic surgery and $80 \%$ for nonsurgical retreatment, but demonstrated no significant difference between the two treatment modalities in a follow up period of more than 4 years. Torabinejad et al(26) conducted a meta-analysis and found that at 2-4 years, endodontic surgery had a higher success rate compared to nonsurgical retreatment; whereas at 4-6 years, nonsurgical retreatment exhibited a higher success rate. The outcomes of this meta-analysis make for a difficult comparison because these time intervals cannot be controlled for in terms of comparison of the same population or the same clinical protocols. In addition, the conclusions of this meta-analysis relied on 
indirect comparisons between endodontic surgery and nonsurgical retreatment from the articles included as there was only one article meeting their criteria for inclusion which made a direct comparison between the two treatment modalities.

The results from the Torabinejad study were consistent with the randomized clinical trial by Kvist et al(27) who showed that while endodontic surgery resulted in a more favorable initial outcome, there was not a significant systematic difference between the two treatment options. Nevertheless, the validity of the outcomes of this randomized clinical trial may be questionable due to the nature of randomization not allowing for proper selection of cases indicated for surgery versus retreatment. Furthermore, the techniques utilized in these previous studies are outdated, so the outcomes may not be representative of those from more recent years with the advent of modern techniques and technology.

\section{Purpose of This Study}

Previous studies regarding both nonsurgical retreatment or root-end surgery vary widely in their methodology of treatment or time frame of follow up after the procedure. Predictability of the outcome of these procedures is needed not only in the short run but in the long-term outlook to allow for proper decision making. To the authors' best knowledge, most surgical outcome studies neglect to specify whether teeth receiving a root-end surgery have or have not received prior nonsurgical retreatment. This distinction is crucial, as the decision to proceed directly to surgical treatment after failed NSRCT, rather than attempt nonsurgical retreatment, suggests ambiguity in personal value judgement. 
The importance of this analysis lies in giving clinicians a long-term outlook to motivate their clinical decision making when it comes to secondary endodontic treatments. This 7-year retrospective study seeks to compare the outcome of nonsurgical retreatments with that of root-end surgeries performed on teeth without prior nonsurgical retreatments. 


\section{MATERIALS AND METHODS}

Electronic enrollment database and claims records from Delta Dental of Wisconsin Insurance were used to acquire the data for this study. The database is the same one utilized in Yavorek et al(28), representing 13,329,249 patient encounters between January 1, 2008 to December 31, 2017. For this study, 1021 teeth of 987 patients who had nonsurgical root canal treatment (NSRCT) were analyzed. Only patients 18 years old and over were included in the study. The triggering event for inclusion in this analysis was identified based on the Code on Dental Procedures and Nomenclature (CDT) codes for nonsurgical root canal therapy categorized as anterior NSRCT (D3310), premolar NSRCT (D3320), and molar NSRCT (D3330).

Only teeth with evidence of primary NSRCT were followed. Any teeth that presented with a code for nonsurgical retreatment or root-end surgery but did not have a prior code for primary NSRCT under this insurance database within the specified time frame were eliminated. For purposes of this study, the teeth tracked were those that specifically had root-end surgery after a failed NSRCT without nonsurgical retreatment performed in between. The only providers included for nonsurgical retreatment or rootend surgery were endodontists.

Survival time was considered from the time of completion of nonsurgical retreatment or root-end surgery to the time of an untoward event. Untoward events were defined as extraction/ root-end surgery after failed nonsurgical retreatment and extraction after failed root-end surgery. Teeth with NSRCT were therefore followed for the presence of CDT codes representing extraction (D7140, D7210), nonsurgical retreatment (D3346, D3347, D3348) or root-end surgery (D3410, D3421, D3425). 
Analyses were performed using SAS 9.4 software (SAS Institute, Cary, NC) and $\mathrm{R}$ version 3.6.3 by the biostatistics department at the Medical College of Wisconsin. Kaplan Meier plots and survival estimates at each year were provided for each variable interested. Cox proportional hazards regression was used to compare survival distributions between categories and the p-value from score test was obtained. P value of $<.05$ was used throughout the analysis for significance level.

Models were generated to assess differences in the following categories: retreatment type comparing nonsurgical retreatment to root-end surgery, nonsurgical retreatment survival by tooth location and by age groups and root-end surgery survival by tooth location and by age groups. All observations were censored at 7 years due to an inadequate number of cases beyond this time frame. Tooth location was categorized as anterior, premolar and molar with no specification made for maxillary, mandibular or tooth number. 


\section{RESULTS}

After the inclusion and exclusion criteria were applied, 806 teeth treated by endodontists were observed in this study. Of these, 506 teeth had nonsurgical retreatment and 300 had a root-end surgery (Table 1). The survival rate of teeth that received nonsurgical retreatment was $90 \%$ after 2 years, $86.8 \%$ after 4 years and $85 \%$ after 6 years. The survival rate of teeth that received root-end surgery was $93.7 \%$ after 2 years, $90.5 \%$ after 4 years and $88 \%$ after 6 years (Figure 1, Table 2). There was not a statistically significant difference $(\mathrm{p}>0.05)$ between treatment types during the follow up timeframe of this study.

The mean age of the patients in this study was 47 years old ranging from minimum 18 years to 77 years of age. The mean age for nonsurgical retreatment was 45.8 and for root-end surgery 49 years old. Of the patients that received nonsurgical retreatment, $29.2 \%$ were in the age range $18-39$ years old, $31.2 \%$ were in the range $40-49$ years old, $26.5 \%$ were in the range $50-59$ years old, and $13 \%$ were in the rage of 60 plus. Of the patients that received root-end surgery, $23 \%$ were in the age range 18-39 years old, $23.3 \%$ were in the range $40-49$ years old, $31.7 \%$ were in the range $50-59$ years old and $22 \%$ were in the range 60 plus (Table 1). A statistically significant difference was found for nonsurgical retreatment between age group 18-39 years old and group 40-49 years old, as well as between group 18-39 years old and group 50-59 and 60+ $(\mathrm{P}<.05)$ (Figure 3, Table 5, 6). Conversely, no statistically significant difference was found between any age groups for root-end surgery (P>.05) (Figure 5, Table 7, 8).

Comparisons were made between treatment survival both within and between tooth locations (defined as anterior, premolar and molar). Of anterior teeth: 41 (8\%) had 
nonsurgical retreatment and $110(36.7 \%)$ had root-end surgery. Of premolars: 97 (19.2\%) had nonsurgical retreatment and 69 (23\%) had root-end surgery. Of molars: $368(72.7 \%)$ had nonsurgical retreatment and $121(40.3 \%)$ had root-end surgery (Table 1).

When comparing tooth location survival (anterior vs premolar, anterior vs molar, premolar vs. molar) for each treatment type, there was no statistical difference $(p>0.05)$ between nonsurgical retreatment and root-end surgery for any pairwise comparison. Likewise, within each specific tooth location (anterior vs premolar vs molar), there was no statistical difference $(\mathrm{p}>0.05)$ between nonsurgical retreatment and root-end surgery survival rates (Figure 2, 4). 
Table 1: Population Data Stratified by Retreatment Type

\begin{tabular}{|c|c|c|c|c|c|}
\hline Variable & $\begin{array}{c}\text { All } \\
(n=806)\end{array}$ & $\begin{array}{l}\text { Nonsurgical } \\
\text { Retreatment } \\
\quad(n=506)\end{array}$ & $\begin{array}{l}\text { Root-End } \\
\text { Surgery } \\
(\mathrm{n}=300)\end{array}$ & p-value & Test \\
\hline Age at NSRCT & & & & $<0.001$ & $\begin{array}{l}\text { Wilcoxon } \\
\text { rank-sum }\end{array}$ \\
\hline Mean (SD) & $\begin{array}{c}47.01 \\
(12.26)\end{array}$ & $45.81(11.67)$ & $\begin{array}{c}49.04 \\
(12.95)\end{array}$ & & \\
\hline $\begin{array}{r}\text { Median } \\
{[\text { Min, Max] }}\end{array}$ & $\begin{array}{c}48.00[18.00 \\
77.00]\end{array}$ & $\begin{array}{c}46.00[18.00 \\
76.00]\end{array}$ & $\begin{array}{c}51.00[18.00 \\
77.00]\end{array}$ & & \\
\hline Freq Missing & 0 & 0 & 0 & & \\
\hline Age at NSRCT & & & & $<0.001$ & Chi-squared \\
\hline $18-39$ & $217(26.9 \%)$ & $148(29.2 \%)$ & $69(23.0 \%)$ & & \\
\hline $40-49$ & $228(28.3 \%)$ & $158(31.2 \%)$ & $70(23.3 \%)$ & & \\
\hline $50-59$ & $229(28.4 \%)$ & $134(26.5 \%)$ & $95(31.7 \%)$ & & \\
\hline $60+$ & $132(16.4 \%)$ & $66(13.0 \%)$ & $66(22.0 \%)$ & & \\
\hline Freq Missing & 0 & 0 & 0 & & \\
\hline Tooth location & & & & $<0.001$ & Chi-squared \\
\hline Anterior & $151(18.7 \%)$ & $41(8.1 \%)$ & $110(36.7 \%)$ & & \\
\hline Molar & $489(60.7 \%)$ & $368(72.7 \%)$ & $121(40.3 \%)$ & & \\
\hline Pre-molar & $166(20.6 \%)$ & $97(19.2 \%)$ & $69(23.0 \%)$ & & \\
\hline
\end{tabular}


Figure 1: Comparison of Tooth Survival Between Nonsurgical Retreatment and Root-End Surgery

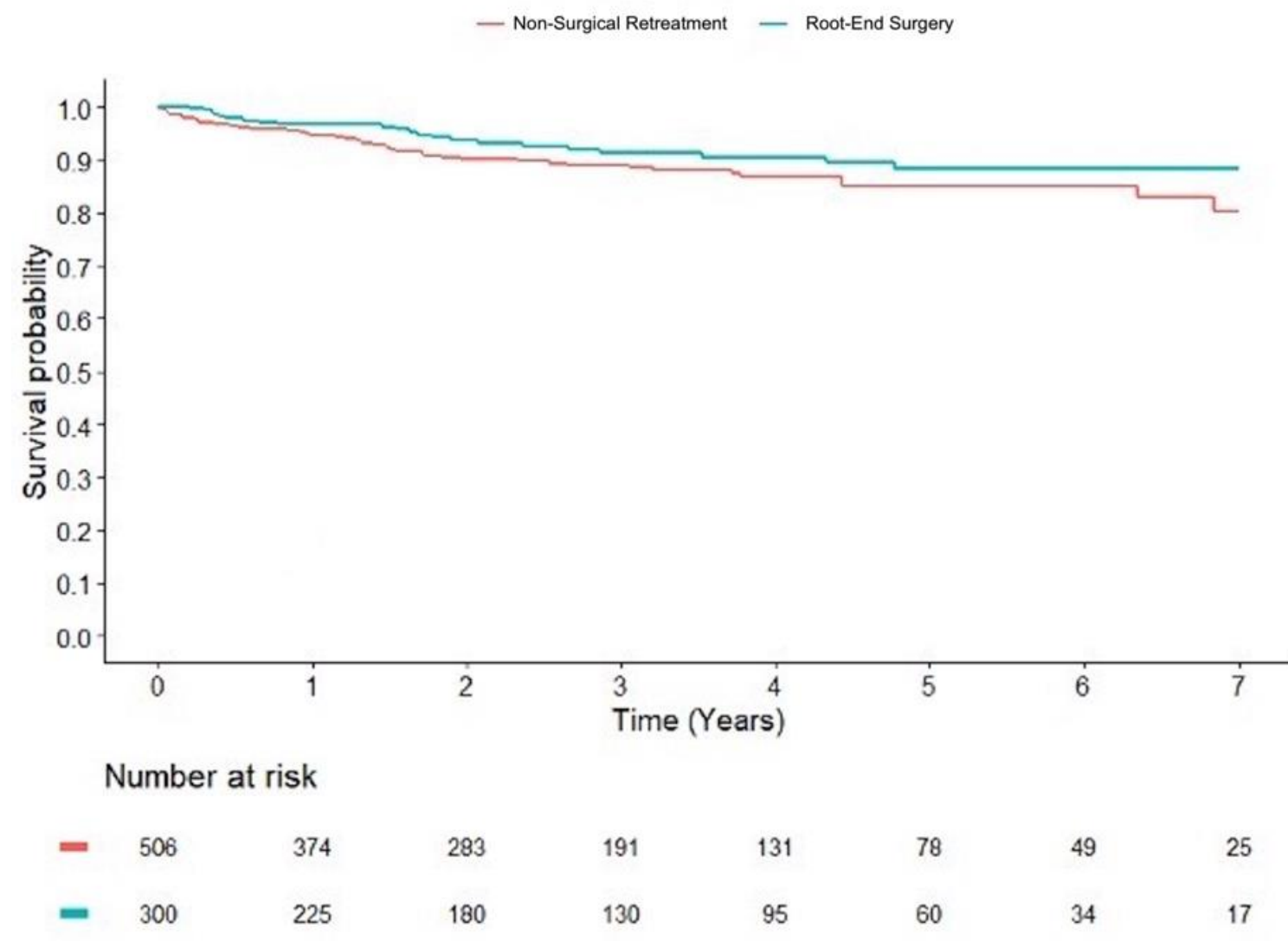


Table 2: Survival Rates for Nonsurgical Retreatment and Root-End Surgery

\begin{tabular}{|ccr|}
\hline Year & Survival & N at risk \\
\hline Nonsurgical Retreatment & $100.00 \%[100.00 \%, 100.00 \%]$ & 506 \\
0 & $94.57 \%[91.91 \%, 96.37 \%]$ & 374 \\
1 & $90.08 \%[86.63 \%, 92.67 \%]$ & 283 \\
2 & $88.96 \%[85.28 \%, 91.77 \%]$ & 191 \\
3 & $86.82 \%[82.52 \%, 90.12 \%]$ & 131 \\
4 & $85.11 \%[80.10 \%, 88.94 \%]$ & 78 \\
5 & $85.11 \%[80.10 \%, 88.94 \%]$ & 49 \\
6 & & \\
Root-End Surgery & & 300 \\
0 & $100.00 \%[100.00 \%, 100.00 \%]$ & 225 \\
1 & $96.61 \%[93.58 \%, 98.23 \%]$ & 180 \\
2 & $93.74 \%[89.78 \%, 96.20 \%]$ & 130 \\
3 & $91.32 \%[86.57 \%, 94.45 \%]$ & 95 \\
4 & $90.50 \%[85.41 \%, 93.88 \%]$ & 60 \\
5 & $88.20 \%[81.92 \%, 92.40 \%]$ & 34 \\
6 & $88.20 \%[81.92 \%, 92.40 \%]$ & \\
\hline
\end{tabular}


Figure 2: Nonsurgical Retreatment Outcomes Categorized by Tooth Location

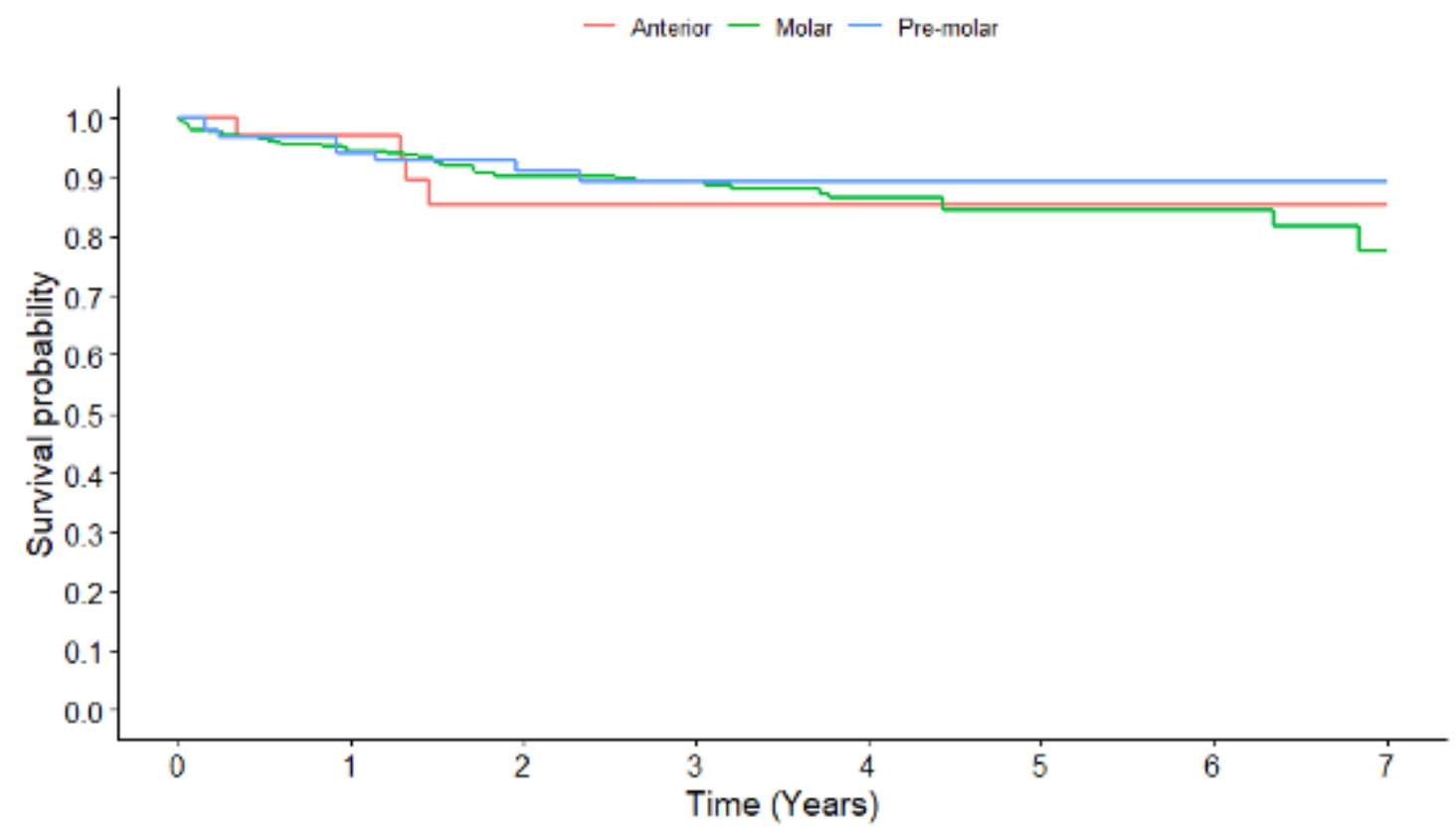

Number at risk

\begin{tabular}{|c|c|c|c|c|c|c|}
\hline 41 & 29 & 18 & 13 & 8 & 5 & 3 \\
\hline 368 & 275 & 213 & 149 & 105 & 59 & 36 \\
\hline 97 & 70 & 52 & 29 & 18 & 14 & 10 \\
\hline
\end{tabular}


Table 3: Nonsurgical Retreatment Survival Rate by Tooth Type

\begin{tabular}{|c|c|c|}
\hline Year & Survival & $\mathrm{N}$ at risk \\
\hline \multicolumn{3}{|c|}{ Anterior } \\
\hline 0 & $100.00 \%[100.00 \%, 100.00 \%]$ & 41 \\
\hline 1 & $96.97 \%[80.31 \%, 99.57 \%]$ & 29 \\
\hline 2 & $85.44 \%[65.13 \%, 94.39 \%]$ & 18 \\
\hline 3 & $85.44 \%[65.13 \%, 94.39 \%]$ & 13 \\
\hline 4 & $85.44 \%[65.13 \%, 94.39 \%]$ & 8 \\
\hline 5 & $85.44 \%[65.13 \%, 94.39 \%]$ & 5 \\
\hline 6 & $85.44 \%[65.13 \%, 94.39 \%]$ & 3 \\
\hline \multicolumn{3}{|c|}{ Molar } \\
\hline 0 & $100.00 \%[100.00 \%, 100.00 \%]$ & 368 \\
\hline 1 & $94.43 \%[91.39 \%, 96.42 \%]$ & 275 \\
\hline 2 & $90.24 \%[86.26 \%, 93.11 \%]$ & 213 \\
\hline 3 & $89.21 \%[84.97 \%, 92.31 \%]$ & 149 \\
\hline 4 & $86.52 \%[81.43 \%, 90.30 \%]$ & 105 \\
\hline 5 & $84.32 \%[78.24 \%, 88.82 \%]$ & 59 \\
\hline 6 & $84.32 \%[78.24 \%, 88.82 \%]$ & 36 \\
\hline \multicolumn{3}{|c|}{ Pre-molar } \\
\hline 0 & $100.00 \%[100.00 \%, 100.00 \%]$ & 97 \\
\hline 1 & $94.10 \%[83.72 \%, 97.94 \%]$ & 70 \\
\hline 2 & $90.99 \%[80.17 \%, 96.05 \%]$ & 52 \\
\hline 3 & $89.09 \%[77.68 \%, 94.86 \%]$ & 29 \\
\hline 4 & $89.09 \%[77.68 \%, 94.86 \%]$ & 18 \\
\hline 5 & $89.09 \%[77.68 \%, 94.86 \%]$ & 14 \\
\hline 6 & $89.09 \%[77.68 \%, 94.86 \%]$ & 10 \\
\hline
\end{tabular}

Table 4: Pairwise Tooth Type Comparison for Nonsurgical Retreatment

\begin{tabular}{|cccc|}
\hline contrast & HR & $95 \%$ CI & p-value \\
\hline Anterior vs Molar & 1.04 & {$[0.3,3.62]$} & 0.996 \\
Anterior vs (Pre-molar) & 1.3 & {$[0.28,5.93]$} & 0.915 \\
Molar vs (Pre-molar) & 1.24 & {$[0.46,3.36]$} & 0.866 \\
\hline
\end{tabular}


Figure 3: Nonsurgical Retreatment Outcomes Categorized by Age Groups

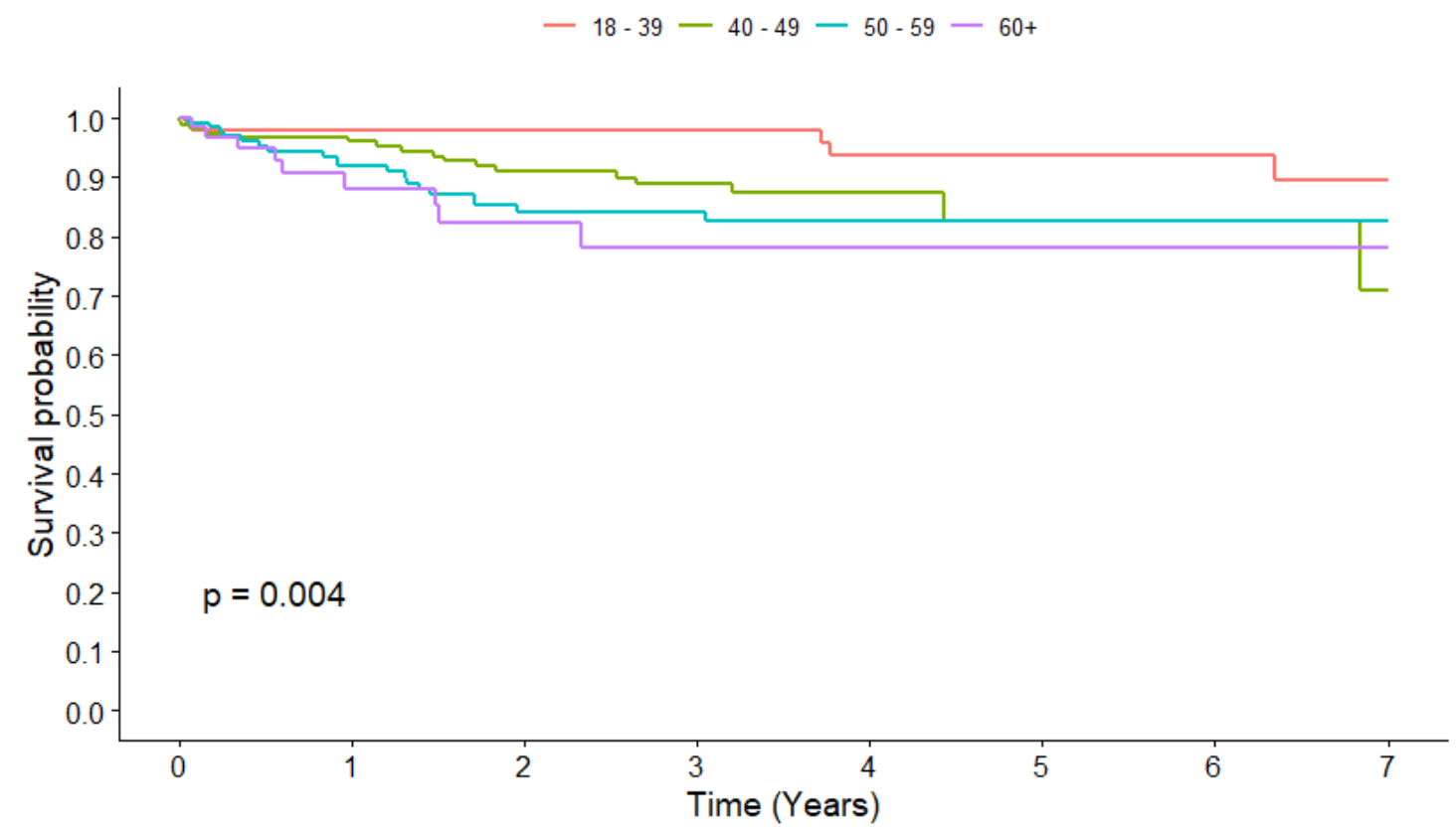

Number at risk

\begin{tabular}{|c|c|c|c|c|c|c|c|}
\hline - & 148 & 110 & 87 & 58 & 42 & 30 & 25 \\
\hline$=$ & 158 & 124 & 95 & 71 & 49 & 25 & 10 \\
\hline$=$ & 134 & 105 & 77 & 51 & 34 & 20 & 11 \\
\hline$=$ & 66 & 35 & 24 & 11 & 6 & 3 & 3 \\
\hline
\end{tabular}


Table 5: Nonsurgical Retreatment Survival Rates for Different Age Groups

\begin{tabular}{|c|c|c|}
\hline Year & Survival & $\mathrm{N}$ at risk \\
\hline \multicolumn{3}{|c|}{$18-39$} \\
\hline 0 & $100.00 \%[100.00 \%, 100.00 \%]$ & 148 \\
\hline 1 & $97.94 \%[93.73 \%, 99.33 \%]$ & 110 \\
\hline 2 & $97.94 \%[93.73 \%, 99.33 \%]$ & 87 \\
\hline 3 & $97.94 \%[93.73 \%, 99.33 \%]$ & 58 \\
\hline 4 & $93.72 \%[83.68 \%, 97.67 \%]$ & 42 \\
\hline 5 & $93.72 \%[83.68 \%, 97.67 \%]$ & 30 \\
\hline 6 & $93.72 \%[83.68 \%, 97.67 \%]$ & 25 \\
\hline \multicolumn{3}{|c|}{$40-49$} \\
\hline 0 & $100.00 \%[100.00 \%, 100.00 \%]$ & 158 \\
\hline 1 & $96.03 \%[91.34 \%, 98.20 \%]$ & 124 \\
\hline 2 & $90.89 \%[84.48 \%, 94.74 \%]$ & 95 \\
\hline 3 & $88.79 \%[81.67 \%, 93.26 \%]$ & 71 \\
\hline 4 & $87.45 \%[79.76 \%, 92.35 \%]$ & 49 \\
\hline 5 & $82.59 \%[71.71 \%, 89.58 \%]$ & 25 \\
\hline 6 & $82.59 \%[71.71 \%, 89.58 \%]$ & 10 \\
\hline \multicolumn{3}{|c|}{$50-59$} \\
\hline 0 & $100.00 \%[100.00 \%, 100.00 \%]$ & 134 \\
\hline 1 & $91.78 \%[84.37 \%, 95.76 \%]$ & 105 \\
\hline 2 & $84.13 \%[75.45 \%, 89.95 \%]$ & 77 \\
\hline 3 & $84.13 \%[75.45 \%, 89.95 \%]$ & 51 \\
\hline 4 & $82.48 \%[73.18 \%, 88.80 \%]$ & 34 \\
\hline 5 & $82.48 \%[73.18 \%, 88.80 \%]$ & 20 \\
\hline 6 & $82.48 \%[73.18 \%, 88.80 \%]$ & 11 \\
\hline \multicolumn{3}{|l|}{$60+$} \\
\hline 0 & $100.00 \%[100.00 \%, 100.00 \%]$ & 66 \\
\hline 1 & $88.15 \%[75.11 \%, 94.59 \%]$ & 35 \\
\hline 2 & $82.27 \%[66.83 \%, 90.99 \%]$ & 24 \\
\hline 3 & $78.16 \%[60.64 \%, 88.57 \%]$ & 11 \\
\hline 4 & $78.16 \%[60.64 \%, 88.57 \%]$ & 6 \\
\hline 5 & $78.16 \%[60.64 \%, 88.57 \%]$ & 3 \\
\hline 6 & $78.16 \%[60.64 \%, 88.57 \%]$ & 3 \\
\hline
\end{tabular}


Table 6: Pairwise Comparison of Age Groups for Nonsurgical Retreatment

\begin{tabular}{|cccc|}
\hline contrast & HR & $95 \%$ CI & p-value \\
\hline$(18-39)$ vs $(40-49)$ & 0.36 & {$[0.11,1.18]$} & 0.117 \\
$(18-39)$ vs $(50-59)$ & 0.28 & {$[0.08,0.92]$} & 0.03 \\
$(18-39)$ vs $(60+)$ & 0.2 & {$[0.05,0.78]$} & 0.013 \\
$(40-49)$ vs $(50-59)$ & 0.78 & {$[0.33,1.84]$} & 0.874 \\
$(40-49)$ vs $(60+)$ & 0.57 & {$[0.2,1.62]$} & 0.509 \\
$(50-59)$ vs $(60+)$ & 0.73 & {$[0.25,2.15]$} & 0.879 \\
\hline
\end{tabular}

Figure 4: Root-End Surgery Outcomes Categorized by Tooth Location

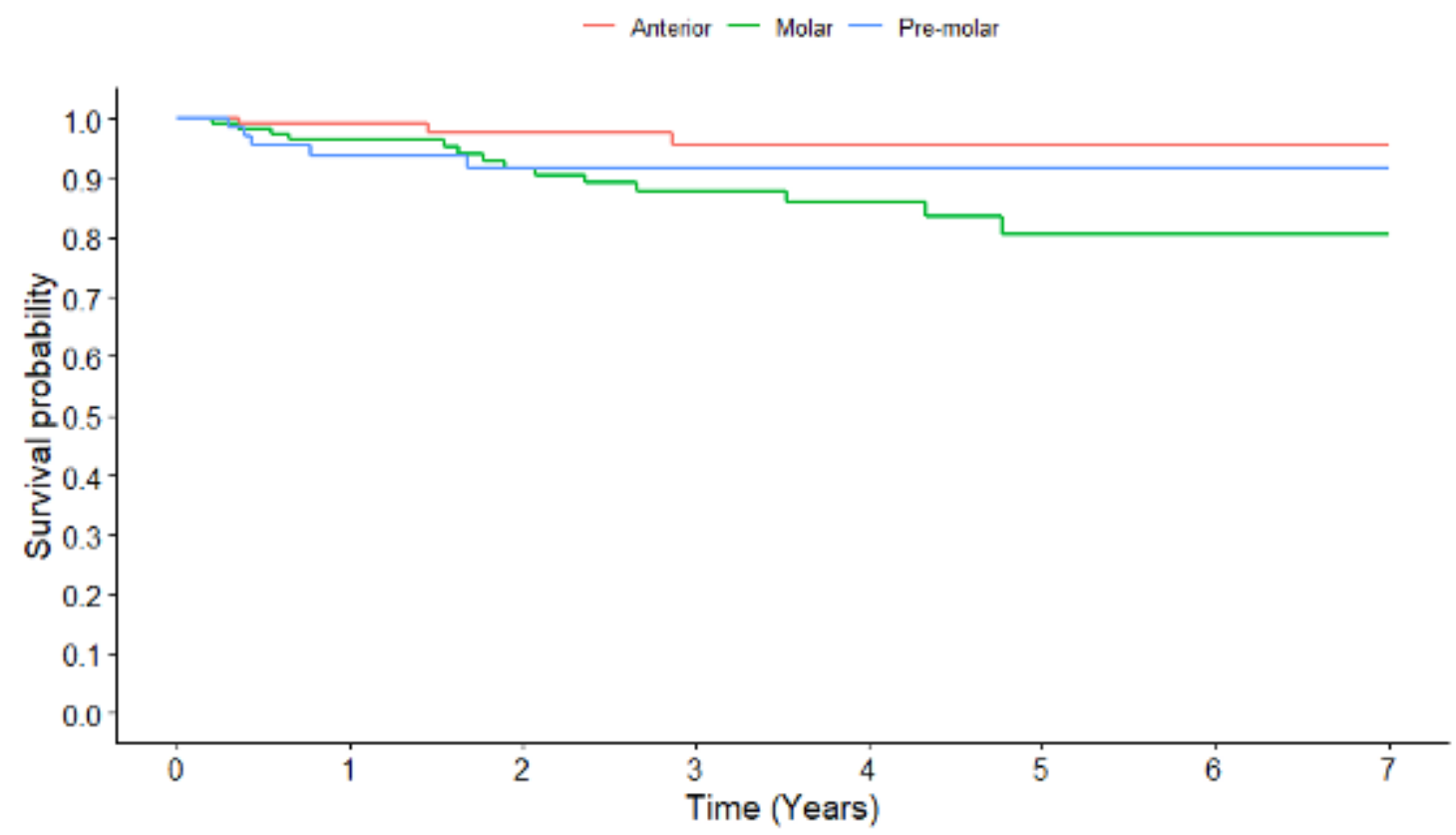

Number at risk

\begin{tabular}{|c|c|c|c|c|c|c|}
\hline 110 & 82 & 64 & 43 & 37 & 27 & 14 \\
\hline 121 & 91 & 76 & 57 & 38 & 22 & 14 \\
\hline 69 & 52 & 40 & 30 & 20 & 11 & 6 \\
\hline
\end{tabular}


Table 7: Root-End Surgery Survival Rate by Tooth Type

\begin{tabular}{|c|c|c|}
\hline Year & Survival & $\mathrm{N}$ at risk \\
\hline \multicolumn{3}{|c|}{ Anterior } \\
\hline 0 & $100.00 \%[100.00 \%, 100.00 \%]$ & 110 \\
\hline 1 & $98.97 \%[92.89 \%, 99.85 \%]$ & 82 \\
\hline 2 & $97.70 \%[91.02 \%, 99.43 \%]$ & 64 \\
\hline 3 & $95.53 \%[85.91 \%, 98.63 \%]$ & 43 \\
\hline 4 & $95.53 \%[85.91 \%, 98.63 \%]$ & 37 \\
\hline 5 & $95.53 \%[85.91 \%, 98.63 \%]$ & 27 \\
\hline 6 & $95.53 \%[85.91 \%, 98.63 \%]$ & 14 \\
\hline \multicolumn{3}{|c|}{ Molar } \\
\hline 0 & $100.00 \%[100.00 \%, 100.00 \%]$ & 121 \\
\hline 1 & $96.28 \%[90.37 \%, 98.59 \%]$ & 91 \\
\hline 2 & $91.73 \%[84.06 \%, 95.80 \%]$ & 76 \\
\hline 3 & $87.82 \%[78.92 \%, 93.12 \%]$ & 57 \\
\hline 4 & $85.91 \%[76.11 \%, 91.90 \%]$ & 38 \\
\hline 5 & $80.41 \%[67.53 \%, 88.59 \%]$ & 22 \\
\hline 6 & $80.41 \%[67.53 \%, 88.59 \%]$ & 14 \\
\hline \multicolumn{3}{|c|}{ Pre-molar } \\
\hline 0 & $100.00 \%[100.00 \%, 100.00 \%]$ & 69 \\
\hline 1 & $93.74 \%[84.11 \%, 97.61 \%]$ & 52 \\
\hline 2 & $91.70 \%[81.02 \%, 96.50 \%]$ & 40 \\
\hline 3 & $91.70 \%[81.02 \%, 96.50 \%]$ & 30 \\
\hline 4 & $91.70 \%[81.02 \%, 96.50 \%]$ & 20 \\
\hline 5 & $91.70 \%[81.02 \%, 96.50 \%]$ & 11 \\
\hline 6 & $91.70 \%[81.02 \%, 96.50 \%]$ & 6 \\
\hline
\end{tabular}


Table 8: Pairwise Tooth Type Comparison for Root-End Surgery

\begin{tabular}{|cccc|}
\hline contrast & HR & $95 \%$ CI & p-value \\
\hline Anterior vs Molar & 0.25 & {$[0.06,1.11]$} & 0.075 \\
Anterior vs (Pre-molar) & 0.39 & {$[0.07,2.19]$} & 0.405 \\
Molar vs (Pre-molar) & 1.56 & {$[0.46,5.31]$} & 0.668 \\
\hline
\end{tabular}

Figure 5: Root-End Surgery Outcomes Categorized by Age Groups

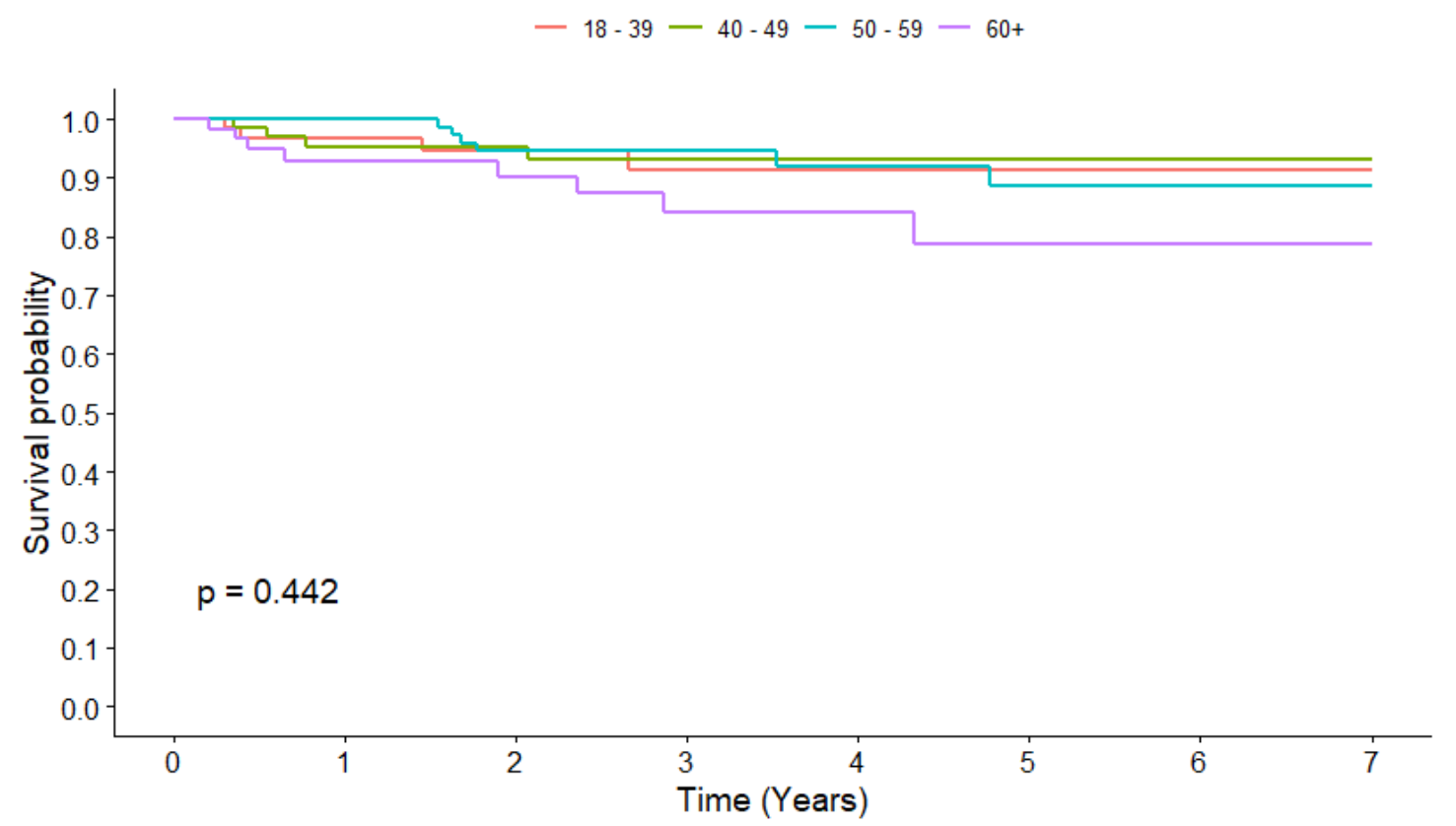

$\begin{array}{ccr} & \text { Number at risk } \\ = & 69 & 47 \\ = & 70 & 5 \\ = & 95 & 80 \\ = & 66 & 40\end{array}$

$\begin{array}{ll}47 & 39 \\ 58 & 47 \\ 80 & 60 \\ 40 & 34\end{array}$

$\begin{array}{ll}39 & 25 \\ 47 & 34 \\ 60 & 48 \\ 34 & 23\end{array}$

$\begin{array}{ll}25 & 18 \\ 34 & 29 \\ 48 & 31 \\ 23 & 17\end{array}$

$\begin{array}{ll}18 & 12 \\ 29 & 14 \\ 31 & 22 \\ 17 & 12\end{array}$

$\begin{array}{ccc}12 & 7 & 4 \\ 14 & 7 & 2 \\ 22 & 13 & 7 \\ 12 & 7 & 4\end{array}$


Table 9: Root-End Surgery Survival Rates for Different Age Groups

\begin{tabular}{|c|c|c|}
\hline Year & Survival & $\mathrm{N}$ at risk \\
\hline \multicolumn{3}{|c|}{$18-39$} \\
\hline 0 & $100.00 \%[100.00 \%, 100.00 \%]$ & 69 \\
\hline 1 & $96.84 \%[87.93 \%, 99.20 \%]$ & 47 \\
\hline 2 & $94.69 \%[84.17 \%, 98.29 \%]$ & 39 \\
\hline 3 & $91.31 \%[77.19 \%, 96.86 \%]$ & 25 \\
\hline 4 & $91.31 \%[77.19 \%, 96.86 \%]$ & 18 \\
\hline 5 & $91.31 \%[77.19 \%, 96.86 \%]$ & 12 \\
\hline 6 & $91.31 \%[77.19 \%, 96.86 \%]$ & 7 \\
\hline \multicolumn{3}{|c|}{$40-49$} \\
\hline 0 & $100.00 \%[100.00 \%, 100.00 \%]$ & 70 \\
\hline 1 & $95.31 \%[86.13 \%, 98.46 \%]$ & 58 \\
\hline 2 & $95.31 \%[86.13 \%, 98.46 \%]$ & 47 \\
\hline 3 & $93.23 \%[82.79 \%, 97.43 \%]$ & 34 \\
\hline 4 & $93.23 \%[82.79 \%, 97.43 \%]$ & 29 \\
\hline 5 & $93.23 \%[82.79 \%, 97.43 \%]$ & 14 \\
\hline 6 & $93.23 \%[82.79 \%, 97.43 \%]$ & 7 \\
\hline \multicolumn{3}{|c|}{$50-59$} \\
\hline 0 & $100.00 \%[100.00 \%, 100.00 \%]$ & 95 \\
\hline 1 & $100.00 \%[100.00 \%, 100.00 \%]$ & 80 \\
\hline 2 & $94.50 \%[85.96 \%, 97.90 \%]$ & 60 \\
\hline 3 & $94.50 \%[85.96 \%, 97.90 \%]$ & 48 \\
\hline 4 & $91.94 \%[80.93 \%, 96.72 \%]$ & 31 \\
\hline 5 & $88.54 \%[74.42 \%, 95.10 \%]$ & 22 \\
\hline 6 & $88.54 \%[74.42 \%, 95.10 \%]$ & 13 \\
\hline \multicolumn{3}{|l|}{$60+$} \\
\hline 0 & $100.00 \%[100.00 \%, 100.00 \%]$ & 66 \\
\hline 1 & $92.68 \%[81.54 \%, 97.20 \%]$ & 40 \\
\hline 2 & $90.10 \%[77.51 \%, 95.83 \%]$ & 34 \\
\hline 3 & $84.01 \%[68.51 \%, 92.29 \%]$ & 23 \\
\hline 4 & $84.01 \%[68.51 \%, 92.29 \%]$ & 17 \\
\hline 5 & $78.76 \%[59.56 \%, 89.58 \%]$ & 12 \\
\hline 6 & $78.76 \%[59.56 \%, 89.58 \%]$ & 7 \\
\hline
\end{tabular}


Table 10: Pairwise Comparison of Age Groups for Root-End Surgery

\begin{tabular}{|cccc|}
\hline contrast & HR & $95 \%$ CI & p-value \\
\hline$(18-39)$ vs $(40-49)$ & 1.2 & {$[0.19,7.54]$} & 0.994 \\
$(18-39)$ vs $(50-59)$ & 1.09 & {$[0.21,5.67]$} & 0.999 \\
$(18-39)$ vs $(60+)$ & 0.46 & {$[0.09,2.21]$} & 0.578 \\
$(40-49)$ vs $(50-59)$ & 0.91 & {$[0.17,4.76]$} & 0.999 \\
$(40-49)$ vs $(60+)$ & 0.38 & {$[0.08,1.85]$} & 0.398 \\
$(50-59)$ vs $(60+)$ & 0.42 & {$[0.11,1.63]$} & 0.353 \\
\hline
\end{tabular}




\section{DISCUSSION}

The purpose of this retrospective study was to compare the outcomes of nonsurgical retreatment with those of root-end surgery after a failed NSRCT and to determine if one treatment option offers a more favorable outcome. Specifically, this study sought to report survival of root-end surgery treated teeth without prior nonsurgical retreatment. This distinction reflects the reality that some practitioners directly proceed to root-end surgery after failed NSRCT, while others pursue nonsurgical retreatment.

Endodontic clinicians possess various routes of treating teeth after failed primary treatment. The decision of which path to take is influenced by various factors, many of which have been delineated in existing literature. Nonsurgical retreatment would be a viable option if original anatomy was not altered during NSRCT(21), if tooth restoration allows for proper accessibility and if failure etiology can be identified and improved. Poor composite restorations or crown margins lead to leakage and contamination of the primary endodontic therapy, which can be rectified with sterile nonsurgical retreatment(29). Studies such as the one from Ray and Trope(6) have confirmed the importance of both high quality coronal restorations and root canal treatments when achieving endodontic success. Attention must also be drawn to the role of patient systemic health and healing capacity when treatment planning, a consideration that often marks patients as better candidates for nonsurgical retreatment. It is noteworthy that at times, a combination of nonsurgical retreatment and root-end surgery is necessary.

Root-end surgery may be indicated when the suspected etiology of persistent periradicular disease is unlikely to be resolved by nonsurgical retreatment. Microorganisms can survive in dentinal tubules, canal irregularities, deltas, and isthmuses 
and can be resistant to traditional cleaning and shaping(22). Causes of persistent apical periodontitis may include overextended filling materials, foreign body reactions, periradicular cysts, cholesterol crystals, or apical scar(30). Root-end surgery should also be considered when nonsurgical retreatment is not a practical option. Some teeth that are heavily restored with deep cores or posts can pose greater risks for potential fracture if the entire restoration is deconstructed for a nonsurgical retreatment. Other factors that can make root-end surgery a better option than nonsurgical retreatment include separated instruments, non-negotiable ledges, canal blockages, transportation, aberrant apical anatomy, failure of previous nonsurgical retreatment, cases in which a biopsy is indicated, previously surgerized teeth or teeth with suspected vertical root fracture.

Over the years, advancements in endodontic armamentarium have positively changed the success rate of both nonsurgical retreatments and root-end surgery, showcasing them as reliable treatment options in contemporary endodontics. Diagnostic improvements through the use of CBCT allow for better visualization of tooth morphology, lesion size/location and surrounding anatomical structures. The ability to make accurate measurements in all directions is paramount in proper planning of surgeries. Increased illumination and magnification allow for a more conservative surgical access and enhanced visualization of apical anatomy and root fractures(31). Furthermore, the use of ultrasonics provides a precise approach to cleaning and shaping of the prepared root end(15). While amalgam and zinc oxide eugenol cements were historically used, issues with cytotoxicity, staining, and leakage paved the way toward more biocompatible root-end materials like MTA and bioceramics(32). 
This study found that there was not a statistically significant difference $(p<0.05)$ between the two treatment types, thereby substantiating the findings of Kvist et al(27) and Riis et al(33). The survival results observed among nonsurgical retreatments after 6 years $(85 \%)$ and root-end surgeries $(88 \%)$ were comparable in value with those found in previous studies from Salehrabi(11) and Song(10). There was no association between tooth site and long-term outcome of treatment for neither nonsurgical retreatment nor root-end surgery. These findings suggest that tooth location is not a detrimental factor in survival rate for the aforementioned treatment options.

When analyzing the prevalence of nonsurgical retreatment or root-end surgery for each tooth type, anterior teeth had a higher likelihood of getting root-end surgery $(36.7 \%)$ compared to nonsurgical retreatment $(8.1 \%)$. Conversely, this relationship reversed for molars, with a higher likelihood of them receiving nonsurgical retreatment $(72.7 \%)$ than root-end surgery $(40.3 \%)$. For premolars, the distribution seems more even with $19.2 \%$ receiving nonsurgical retreatment and $23 \%$ receiving root-end surgery. The relationship between anteriors and molars can be explained due to the nature of difficulty of molars with harder surgical access and more complex anatomy(34).

This study also found no statistically significant difference between age groups for root-end surgery. These findings were consistent with those from von Arx et al(12) who reported that even though a slightly higher success rate was observed in patients under the age of 45 compared to those over 45 , no significant difference was found. Regarding nonsurgical retreatment, our findings of a significant difference between the younger and older age groups are not supported by previous studies. Farzaneh et al(20) showed no difference between age groups, concluding that age was not a factor in the 
outcome of nonsurgical retreatment. However, this could be a result of different population cohort in that study versus the present study. In our study, patients in the older age groups of 50 years old and above appeared to receive more root-end surgery compared to the younger age groups who tended to receive more nonsurgical retreatment when comparing the two treatment modalities. Older populations tend to have more heavily restored teeth thus surgery would be a more viable choice.

In this analysis, a follow up of 7 years allowed for a broader view of trends within outcomes of nonsurgical retreatments and root-end surgeries. After the author's stringent inclusion and exclusion criteria were applied, the number of teeth observed was 806 . This number may seem low considering the long time span of the study; however, it confirms the high survival rate of primary root canal therapy(1). The initial intent of the authors was to also evaluate treatment outcomes between different types of providers (general dentists, oral surgeons) in addition to endodontists, but this data was excluded from the analysis due to insufficient sample size. When compared to other studies of this realm, the 7-year timeframe is one of longer follow ups conducted; nevertheless, the nature of an insurance-based study has limitations. The multifactorial variables that affect success of treatment modalities cannot be accounted for in the data collection. Additionally, the insurance database was limited to only one state, denoting regional treatment philosophies that may be different from other areas of the country. Further large-scale studies warrant utilization of a nationwide database to better assess long-term outcomes.

Treating failed NSRCT is complex and requires a holistic approach when performing diagnosis, case selection, and treatment planning. Due to the lack of a statistical significance between nonsurgical retreatment and root-end surgery survival 
rates and no difference in tooth type, the authors emphasize the importance of synthesizing all pertinent factors when making clinical decisions. Knowledge of these treatment outcomes is critical in supporting the decisions made by both practitioners and/or patients. Patient preference, financial situation and/or insurance coverage might also play a role in selecting the next treatment modality. 


\section{CONCLUSION}

Within the constraints and limitations of this study, the survival rate of nonsurgical retreatment and root-end surgery after 6 years was $85 \%$ and $88.5 \%$, respectively, with no significant difference between the two treatment options. Tooth

location was not a determining factor in the survival rate after nonsurgical retreatment or root-end surgery. 


\section{BIBLIOGRAPHY}

1. Salehrabi R, Rotstein I. Endodontic treatment outcomes in a large patient population in the USA: An epidemiological study. J Endod 2004;30:846-50.

2. Lazarski MP, Walker WA 3rd, Flores CM, Schindler WG, Hargreaves KM. Epidemiological evaluation of the outcomes of nonsurgical root canal treatment in a large cohort of insured dental patients. J Endod 2001;27:791-6.

3. Nair PNR. On the causes of persistent apical periodontitis: A review. Int Endod J 2006;39:249-81.

4. Farzaneh M, Abitbol S, Lawrence HP, Friedman S. Treatment outcome in endodontics - The Toronto study. Phase II: Initial treatment. J Endod 2004;30:3029.

5. Yee K, Bhagavatula P, Stover S, et al. Survival Rates of Teeth with Primary Endodontic Treatment after Core/Post and Crown Placement. J Endod 2018;44:220-5.

6. Ray Ha, Trope M. Periapical status of endodontically treated teeth in relation to the technical quality of the root filling and the coronal restoration. Int Endod J $1995 ; 28: 12-8$.

7. Burry JC, Stover S, Eichmiller F, Bhagavatula P. Outcomes of Primary Endodontic Therapy Provided by Endodontic Specialists Compared with Other Providers. J Endod 2016;42:702-5.

8. Moreira MS, Anuar ASNS, Tedesco TK, dos Santos M, Morimoto S. Endodontic Treatment in Single and Multiple Visits: An Overview of Systematic Reviews. J Endod 2017;43:864-70.

9. Manfredi M, Figini L, Gagliani M, Lodi G. Single versus multiple visits for endodontic treatment of permanent teeth. Cochrane Database Syst Rev 2016;12.

10. Song M, Chung W, Lee SJ, Kim E. Long-term outcome of the cases classified as successes based on short-term follow-up in endodontic microsurgery. J Endod 2012;38:1192-6.

11. Salehrabi R, Rotstein I. Epidemiologic Evaluation of the Outcomes of Orthograde Endodontic Retreatment. J Endod 2010;36:790-2.

12. von Arx T, Jensen SS, Hänni S. Clinical and radiographic assessment of various predictors for healing outcome 1 year after periapical surgery. J Endod. 2007 Feb;33:123-8. 
13. Zuolo ML, Ferreira MO, Gutmann JL. Prognosis in periradicular surgery: a clinical prospective study. Int Endod J. 2000 Mar;33:91-8.

14. Tsesis I, Rosen E, Taschieri S, Telishevsky Strauss Y, Ceresoli V, Del Fabbro M. Outcomes of surgical endodontic treatment performed by a modern technique: an updated meta-analysis of the literature. J Endod. 2013 Mar;39:332-9.

15. Tsesis I, Rosen E, Schwartz-Arad D, Fuss Z. Retrospective evaluation of surgical endodontic treatment: traditional versus modern technique. J Endod. 2006 May;32:412-6.

16. Kim S, Kratchman S. Modern endodontic surgery concepts and practice: a review. J Endod. 2006 Jul;32:601-23.

17. Shinbori N, Grama AM, Patel Y, Woodmansey K, He J. Clinical outcome of endodontic microsurgery that uses EndoSequence BC root repair material as the root-end filling material. J Endod. 2015 May;41:607-12.

18. Rubinstein RA, Kim S. Short-term observation of the results of endodontic surgery with the use of a surgical operation microscope and super-EBA as root-end filling material.

J Endod 1999;25:43-8.

19. Rubinstein RA, Kim S. Long-term follow-up of cases considered healed one year after apical microsurgery. J Endod. 2002 May;28:378-83.

20. Farzaneh M, Abitbol S, Friedman S. Treatment outcome in endodontics: the Toronto study. Phases I and II: Orthograde retreatment. J Endod. 2004 Sep;30:62733.

21. Gorni FG, Gagliani MM. The outcome of endodontic retreatment: a 2-yr followup. J Endod. 2004 Jan;30:1-4.

22. Sundqvist G, Figdor D, Persson S, et al. Microbiologic analysis of teeth with failed endodontic treatment and the outcome of conservative re-treatment. Oral Surg Oral Med Oral Pathol Oral Radiol Endod 1998;85:86-93.

23. Chugal NM, Clive JM, Spångberg LS. A prognostic model for assessment of the outcome of endodontic treatment: Effect of biologic and diagnostic variables. Oral Surg Oral Med Oral Pathol Oral Radiol Endod. 2001 Mar;91:342-52.

24. Imura N, Pinheiro ET, Gomes BP, Zaia AA, Ferraz CC, Souza-Filho FJ. The outcome of endodontic treatment: a retrospective study of 2000 cases performed by a specialist. J Endod. 2007 Nov;33):1278-82. 
25. Kang M, In Jung H, Song M, Kim SY, Kim HC, Kim E. Outcome of nonsurgical retreatment and endodontic microsurgery: a meta-analysis. Clin Oral Investig. 2015 Apr;19:569-82.

26. Torabinejad M, Corr R, Handysides R, Shabahang S. Outcomes of nonsurgical retreatment and endodontic surgery: a systematic review. J Endod. 2009 Jul;35:930-7.

27. Kvist T, Reit C. Results of endodontic retreatment: a randomized clinical study comparing surgical and nonsurgical procedures. J Endod. 1999 Dec;25:814-7.

28. Yavorek A, Bhagavatula P, Patel K, Szabo A, Ibrahim M. The Incidence of Root Canal Therapy after Full-Coverage Restorations: A 10-Year Retrospective Study. J Endod. 2020 May;46:605-10.

29. Gillen BM, Looney SW, Gu LS, Loushine BA, Weller RN, Loushine RJ, Pashley $\mathrm{DH}$, Tay FR. Impact of the quality of coronal restoration versus the quality of root canal fillings on success of root canal treatment: a systematic review and metaanalysis. J Endod. 2011 Jul;37:895-902.

30. Nair PN. Pathogenesis of apical periodontitis and the causes of endodontic failures. Crit Rev Oral Biol Med. 2004 Nov 1;15:348-81.

31. Setzer FC, Kohli MR, Shah SB, Karabucak B, Kim S. Outcome of endodontic surgery: a meta-analysis of the literature--Part 2: Comparison of endodontic microsurgical techniques with and without the use of higher magnification. $\mathrm{J}$ Endod. 2012 Jan;38:1-10.

32. Torabinejad M, Watson TF, Pitt Ford TR. Sealing ability of a mineral trioxide aggregate when used as a root end filling material. J Endod. 1993 Dec;19:591-5.

33. Riis A, Taschieri S, Del Fabbro M, Kvist T. Tooth Survival after Surgical or Nonsurgical Endodontic Retreatment: Long-term Follow-up of a Randomized Clinical Trial. J Endod. 2018 Oct;44:1480-6.

34. Kim S, Jung H, Kim S, Shin SJ, Kim E. The Influence of an Isthmus on the Outcomes of Surgically Treated Molars: A Retrospective Study. J Endod. 2016 Jul;42:1029-34. 